\title{
The Effect of a Lifestyle Intervention Program Using a Mobile Application for Adults with Metabolic Syndrome, versus the Effect of a Program Using a Booklet: A Pilot Randomized Controlled Trial
}

This article was published in the following Dove Press journal:

Clinical Interventions in Aging

\section{Eliza Mi Ling Wong' \\ Doris YP Leung (D) \\ Hon Lon Tam' \\ Qun Wang $^{2}$ \\ Kai Wang Yeung (D) \\ Angela YM Leung (D)}

'School of Nursing, The Hong Kong Polytechnic University, Hong Kong, SAR, People's Republic of China; ${ }^{2}$ School of Nursing, Shenzhen University, Shenzhen, Guangdong, People's Republic of China
Correspondence: Eliza Mi Ling Wong School of Nursing, GH 52I, School of Nursing, The Hong Kong Polytechnic University, Hung Hom, Kowloon,

Hong Kong, SAR, People's Republic of China

Tel $+86852-27666544$

Fax $+86852-23649663$

Email eliza.wong@polyu.edu.hk
Purpose: This study aimed to examine the preliminary effect, feasibility, and acceptability of a lifestyle intervention program using a mobile application (app) versus the effect of a program using a booklet for adults with metabolic syndrome (MetS).

Patients and Methods: This trial was conducted in two community centers of Hong Kong. Participants were included if they were adults with MetS, aged over 50, and able to use a smartphone. Eligible subjects were randomly assigned to either the app group or booklet group. Those in the booklet group received a health talk and a booklet, whereas those in the app group received a health talk and a MetS app to support their exercise maintenance and health records for 3 months. Both groups received similar educational content related to healthcare for MetS clients. Data were collected at baseline (T1) and at 1- (T2) and 3-month (T3) intervals. Outcomes were body weight (primary outcome), total amount of exercise, blood pressure, and lipid concentrations. Data were analyzed using the generalized estimating equation models. Feasibility and acceptability were assessed in process evaluation.

Results: Ninety-eight individuals were screened for eligibility and 77 were randomized into the app group $(\mathrm{n}=38)$ or booklet group $(\mathrm{n}=39)$. The attrition rate at T3 was $11.690 \%$. The app group showed a significant reduction in body weight $(\beta=-1.069, p=0.012)$ and body mass index $(\beta=-0.371, p=0.026)$, a greater amount of exercise $(\beta=8.454, p=0.032)$, and improved exercise self-efficacy $(\beta=10.62, p=0.001)$ within 3 months. There were no significant differences between groups for other outcomes. The participants appreciated the proposed intervention of the programme.

Conclusion: The MetS app may be incorporated in the health promotion programme to support exercise maintenance and a healthy lifestyle in the community.

Keywords: exercise, body weight, metabolic syndrome, intervention, programme, mobile application

\section{Introduction}

Metabolic syndrome (MetS) exists in those who have a cluster of the major heart attack risk factors: diabetes and prediabetes, abdominal obesity, high cholesterol concentrations, and high blood pressure. ${ }^{1}$ According to the new International Diabetes Federation (IDF) definition, a person with MetS has central obesity (defined as a certain waist circumference, which has ethnicity-specific values) and has any two of the following factors, or is receiving treatment for these: 
elevated triglyceride concentrations, reduced HDL cholesterol concentrations, high blood pressure or previously diagnosed hypertension, or elevated plasma glucose concentrations or previously diagnosed type 2 diabetes. ${ }^{1}$ MetS is becoming increasingly prevalent, with a prevalence of $29 \%$ to $40 \%$ in Western countries ${ }^{2,3}$ and approximately $25 \%$ in China, ${ }^{4}$ with an increasing prevalence in young-old adults. ${ }^{5}$ MetS further deteriorates the condition of diabetes and prediabetes, cardiovascular disease, and stroke, if not well treated. ${ }^{1,5,6}$

Lifestyle modifications, such as increasing physical activity, making healthier dietary choices, and giving up smoking, have been recommended as the primary interventions for MetS. ${ }^{1,6,7}$ Previous studies have reported various modalities of lifestyle intervention for MetS management, including educational programs comprising a range of educational sessions, on-site exercise supervision, and home-based lifestyle interventions that include a booklet and follow-up advice over the telephone..$^{8-12}$ However, these programs have been challenging to deliver in a scalable manner to meet the large demand of the MetS population. Little is known about whether the effects of these programs on exercise behavior and other outcomes can be improved by incorporating e-health technology.

E-health technology refers to the use of information and communication technologies to improve health care. ${ }^{13}$ During the past decade, e-health technology using mobile applications (apps) has proved to be an effective mode of delivery for educational interventions. ${ }^{13-21}$ Recent evidence has shown the effectiveness of smartphone apps at increasing physical activity in the short term (eg, up to 3 months). However, most e-health tools are web-based platforms or the apps are designed for the general public and not specifically for MetS clients. Moreover, the lack of continuous selfmonitoring or individual feedback on outcomes seldom results in behavioral changes. ${ }^{16,20-24}$ Hence, a highly interactive e-health mobile app (MetS app) was developed in this study. An effective MetS app should be relevant, feature personalized plans and goal setting, and address specific characteristics that suit clients with MetS (eg, should be selfmanaged, flexible, and interactive). Although recent studies have proposed the use of mobile apps in wellnessmaintenance programs globally and locally ${ }^{16,19,23,24}$ more advanced apps should be designed, applied, and evaluated for patients with specific health problems, such as MetS.

Considering the success of a previously reported homebased lifestyle-intervention program (LIP), ${ }^{12}$ the advantages of e-health programs, ${ }^{13-21}$ and the growing number of young-old (aged 50 or above) adults with MetS, ${ }^{2-5}$ we aimed to develop an e-health app and integrate it into a lifestyle-intervention program. The aims of this study were to examine the preliminary effect of an LIP using a mobile app for young-old adults with MetS versus the effects of an LIP using a booklet. Feasibility and acceptability of the intervention were further assessed in process evaluation. The specific objectives were to examine the effects of these programs on

1. body weight (primary outcome),

2. exercise: total exercise time per week, total amount of exercise,

3. exercise self-efficacy, and

4. MetS profiles (waist circumference, blood pressure, LDL cholesterol [LDL-C] concentrations, HDL cholesterol [HDL-C] concentrations, and triglyceride and blood glucose concentrations).

5. Feasibility and acceptability of the intervention: by examination of recruitment rate, attrition rate and feedback by participants in process evaluation.

We hypothesized that clients receiving an LIP with a tailor-made app (app group) would perform better than those in the control group receiving an LIP with a booklet, in terms of their body weight reduction. The app group would have better engagement in exercise and exercise self-efficacy, leading to improved control of their MetS risk factor profile (waist circumference, blood pressure, LDL-C and HDL-C concentrations, triglyceride concentrations, and glycemic control) within 3 months.

\section{Materials and Methods Design}

The study used a prospective pilot randomized controlled trial (RCT) design, with randomization to either the intervention (the app group) or control group (the booklet group). This design was guided by the Consolidated Standards of Reporting Trials checklist. ${ }^{25}$ The study was conducted from April 2018 to March 2019.

\section{Study Setting, Subjects, and Sample Size}

The study was conducted in two community centers of Hong Kong. The community centres that regularly hold various health seminars and activities for members living nearby were used for subject recruitment. Clients voluntarily registered for our project after reading the promotional 
poster. By specific appointment, subject recruitment and follow-up visits were held in the community centers or the university research center.

\section{Inclusion Criteria}

Individuals who satisfied the following inclusion criteria were invited to participate in the RCT. Individuals were included if they were

1. ethnic Chinese;

2. owned a smartphone;

3. aged 50 or above; and

4. had MetS, ${ }^{1}$ as defined by central obesity (waist circumference: male $>90 \mathrm{~cm}$, female $>80 \mathrm{~cm}$ ) and two of the following:

- triglyceride concentrations $\geq 150 \mathrm{mg} / \mathrm{dL} \quad(1.7$ $\mathrm{mmol} / \mathrm{L}$ ), or treatment for this lipid abnormality,

- HDL cholesterol $<40 \mathrm{mg} / \mathrm{dL}(1.03 \mathrm{mmol} / \mathrm{L})$ in males and $<50 \mathrm{mg} / \mathrm{dL}(1.29 \mathrm{mmol} / \mathrm{L})$ in females, or treatment for this lipid abnormality,

- systolic blood pressure $\geq 130$ or diastolic blood pressure $\geq 85 \mathrm{mmHg}$, or treatment of previously diagnosed hypertension, or

- fasting plasma glucose $\geq 100 \mathrm{mg} / \mathrm{dL}$ (5.6 mmol/L) or previously diagnosed type 2 diabetes.

\section{Exclusion Criteria}

Individuals with physical health problems; mental, visual, or cognitive impairments that compromised their use of a smartphone; or contraindications to walking or performing exercise were excluded.

\section{Sample Size}

For a pilot study, Hertzog ${ }^{26}$ suggested that at least 30 participants should be recruited per group. Eventually, we recruited 38 participants in the intervention group and 39 participants in the control group after taking into consideration an $18 \%$ attrition rate at 3 months of follow-up, as reported by Wang et al. ${ }^{12}$

\section{Randomization and Masking}

Eligible participants were randomly allocated to either the app group or the booklet group. The allocation sequence was computer-generated by a statistician, and blinded to the investigators. Participants were randomized at a ratio of 1:1. A small card indicating the group assignment was placed in a sealed opaque envelope and a research assistant (RA) opened the envelope after a participant had completed the baseline questionnaire. The outcome assessor, who was the nurse who gave the talks, was blinded to the group assignment. In addition, different follow-up dates were assigned to the two groups to avoid subject contamination.

\section{Intervention}

\section{Standard Care}

All participants of both groups received a 30-min educational session related to MetS, which was conducted by a nurse. The health educational session comprised a group of 8-12 participants and was held in a community center. The LIP educational session was conducted by a nurse and the content (educational session and booklet) was similar to the content used by Wang et al, except for a minor modification on dietary suggestions to suit the Hong Kong culture. $^{12}$ The educational content covered the knowledge related to metabolic syndrome, risk factors and its medical care, the importance of lifestyle modification such as exercise, proper diet, quit smoking and stress management. The content of the booklet was further examined by a panel of experts in Hong Kong, comprising a senior nurse, a dietitian, two nursing professors, and a MetS patient. All participants were advised to perform a brisk walking exercise or their usual exercise regimen for at least 30 minutes per day for 5 days per week, as recommended by the World Health Organization (WHO). Regular brisk walking is reported to be a safe exercise that can improve cardiovascular function and muscle strength; improve balance, blood circulation, and mood; and maintain physical and psychological well-being. ${ }^{27,28}$

\section{The Booklet Group}

The booklet group received a health talk plus a booklet that provided an overview of MetS and its risk factors and advice on lifestyle management, a healthy diet for MetS patients, regular exercise, stress management, medication, and self-monitoring of health.

\section{App Group and Theoretical Framework of the App Design}

The app group received a health talk and an instalment of the MetS app. The MetS app aimed to support the initiation and maintenance of behavioral changes, such as self-observation and monitoring of waist circumference and body weight, goal setting, recording and providing feedback on the amount and pattern of exercise. The design of the MetS app was based on the health belief model. ${ }^{29-31}$ In this study, the program, together with the support of the MetS app, attempted to guide the 
participant to achieve the above aims. In line with this construct of perceived susceptibility, severity, benefits, and barriers, the MetS app and booklet provided facts on MetS and its risk factors and the consequences of insufficient physical exercise and poor dietary control, which may lead to the progression of MetS and related complications. In addition, the benefits and positive outcomes of regular exercise were described. The MetS app was installed on a smartphone to provide health information for MetS patients, and also provided an option to support personal health and exercise self-monitoring and management strategies.

In summary, both groups received same educational content by nurse, supplemented by either booklet or app knowledge platform. However, the app provided features to further motivate and support client's individual exercise maintenance (exercise goal setting and input of exercise type and time) and self-monitoring of health record such as input and retrieve the record of body weight, blood pressure, optional blood glucose and lipid level.

\section{Procedure and Data Collection}

Promotional posters were posted in two community centers and on the university notice boards. The health talks were conducted by a nurse as scheduled in the community centers. After the talks, potential study participants were further screened, and approached by a research assistant (RA) and invited to join the study. At the scheduled appointment, a trained RA to further confirm the individual's eligibility performed a health assessment (waist circumference, fasting glucose and lipid concentrations). Eligible participants then signed a written consent form after receiving an explanation of the project. Baseline data (T1) were collected and each eligible participant was randomly assigned to either the app group or the booklet group, after which they received the appropriate intervention according to the protocol. In followup appointments at $\mathrm{T} 2$ and $\mathrm{T} 3$, another RA who was blinded to the group assignment re-collected the data. The blood lipid concentration, LDL-C concentration, and HDL-C concentration data were collected at baseline, T1, and T3 only. The study flow is shown in Figure 1.

\section{Ethical Considerations}

This trial was conducted in accordance with the Declaration of Helsinki. Ethical approval was obtained from Human Subjects Ethics Sub-committee, Hong Kong Polytechnic University. All participants were informed about the purpose and procedure of the study before signing a consent form.
Participants could voluntarily join or withdraw at any time without adverse effects. Confidentiality and anonymity were assured during the study. App safety and data security were closely monitored, according to the university's guidelines for web and app safety.

\section{Measures}

A structured questionnaire was developed to collect demographic data (age, sex, marital status, education, employment), clinical data (history of hypertension, diabetes, dyslipidemia, and smoking), and outcomes. Data were collected at $\mathrm{T} 1, \mathrm{~T} 2$, and $\mathrm{T} 3$.

\section{Primary Outcome}

The primary outcome, body weight, was measured and recorded by the research assistant at $\mathrm{T} 1, \mathrm{~T} 2$, and $\mathrm{T} 3$ at a community center or university research centre using the same weight scale for each measurement.

\section{Secondary Outcomes}

Secondary outcomes were measured at T1, T2, and T3. The total amount of physical exercise was quantified using a modified version of the Godin-Shephard leisure-time physical activity (GSLTPA) questionnaire. ${ }^{32,33}$ The participants were instructed to indicate the amount of exercise they performed and to rank the exercises in order of difficulty (strenuous, moderate, or mild). The total score was then calculated. This approach has been widely used in empirical studies and has been shown to exhibit good psychometric properties. ${ }^{32,33}$ Total exercise time per week was also recorded.

\section{Chinese Version of the Self-Efficacy for Exercise Scale}

The Chinese version of the self-efficacy for exercise (SEEC) scale consists of nine items, and responses are made on a scale of $0-10$. The sum of the responses to all nine items yields the final composite score, which ranges from 0 to 90 . The SEE-C has been shown to be reliable, with good psychometric properties for older Chinese adults in Hong Kong, as indicated by a Cronbach's alpha coefficient of $0.75 .^{34}$

\section{Demographic, Clinical, and Metabolic Syndrome Risk Factor Data}

Demographic data, including age, sex, occupation, educational level, and medical history, including health and drug-use history, were collected by a RA. Blood lipid data, including LDL-C, HDL-C, and triglyceride concentrations were tested by Cobas 101 Lipid testing machine (manufacturer: Roche Diagnostics); blood sugar concentrations; and all other parameters, including waist 


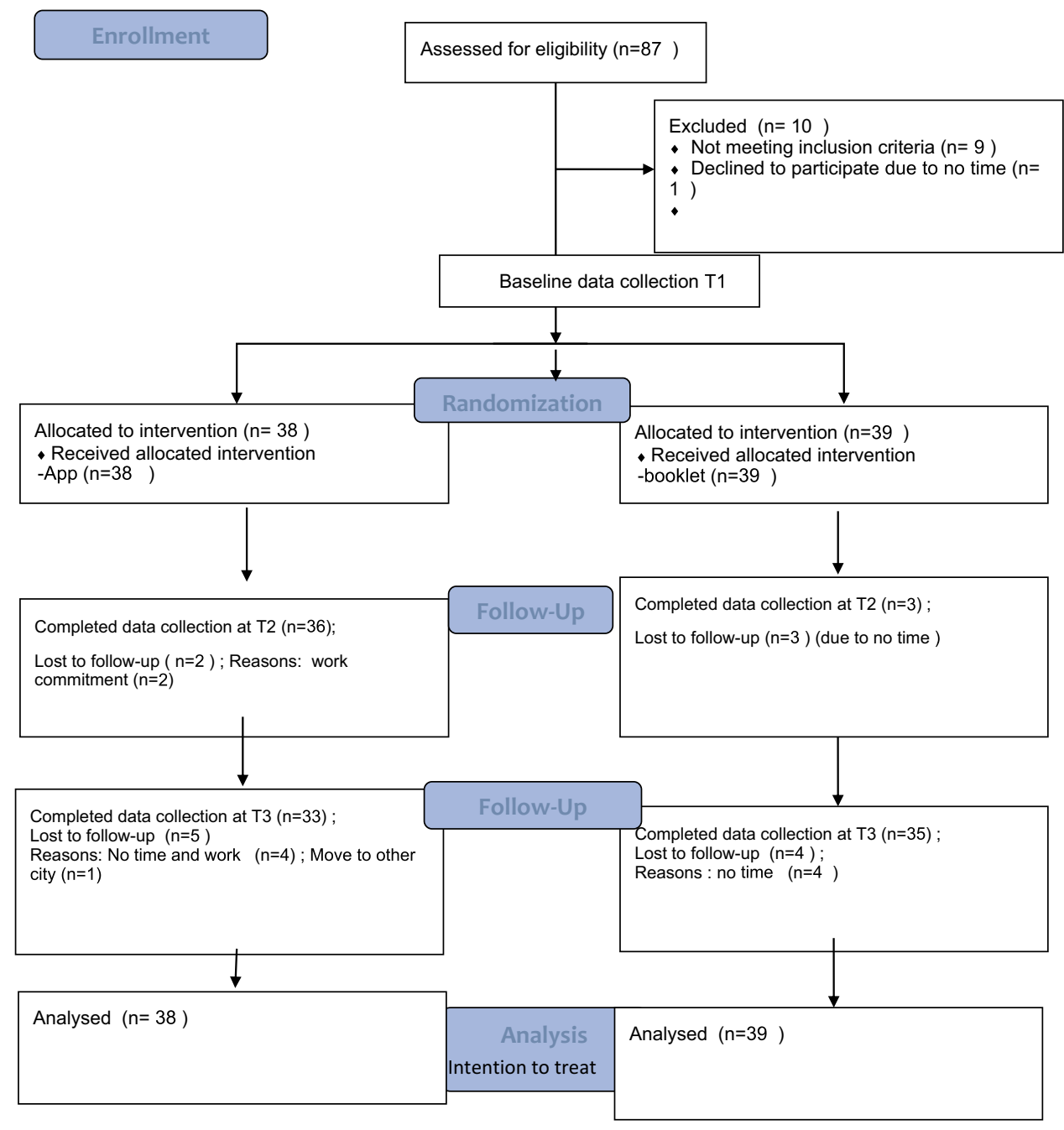

Figure I Study flow chart.

circumference and systolic and diastolic blood pressure, were collected by a RA at $\mathrm{T} 1$ and $\mathrm{T} 3$.

\section{Process Evaluation of Feasibility and Acceptability}

To assess the implementation and acceptability of the program using the app or booklet, three open-ended questions were asked at the T3 follow-up appointment. These questions were related to satisfaction with the program, perceived acceptability of the intervention (booklet or app), and suggestions for improvement of the MetS app or the LIP booklet. The feasibility of the program was assessed by examining the recruitment rate and the attrition rate.

\section{Data Analysis}

Data were entered and analyzed using SPSS 25.0 (IBM, Armonk, NY, USA). Baseline characteristics and blood lipid data were compared between the two groups using a chi-square or Fisher's exact test for categorical variables, and a Student $t$-test or Mann-Whitney test for continuous variables. To compare the changes in the mean values of continuous outcome variables (waist circumference, body weight, total amount of physical exercise, and exercise self-efficacy) between the two groups, the intention-totreat principle and generalized estimating equation (GEE) models. Generalized estimating equations (GEE) models were employed to assess differential change of the outcomes across three time points between the two groups with adjustment for potential confounding factors. The interaction effect (main effect) of the time point dummy variables and group were included in the GEE models to assess the differential change of the outcomes at each time point relative to the baseline between the two groups. GEE model can account for intra-correlated repeated measures 
data and be able to fit various types of data with the use of appropriate link-function. The GEE model is chosen over the linear mixed effects model because the GEE uses sandwich estimator, which produce consistent estimates even if the correlation structure is misspecified, thus is particularly important for pilot study in which the information of the correlation structure is usually lacking. In addition, the GEE model can accommodate missing data caused by dropouts, provided that data are missing at random which is particularly suitable for ITT analysis, without the need for imputation of missing data. ${ }^{35}$ Between-group Cohen's $d$ were calculated to estimate the effect sizes of the app as compared to the booklet on all the outcome measures separately. ${ }^{36}$ All statistical tests were two-sided and a p-value $<0.05$ was considered an indicator of statistical significance.

In process evaluation, all participants are encouraged to provide written feedback at the end of survey at T3 followup. The qualitative data were subject to content analysis to identify supplementary information. ${ }^{37}$ To enhance the credibility of the qualitative data analysis, two researchers who are familiar with qualitative analysis adopted content analysis by identified significant statements, phrases, sentences and commonalities among the qualitative data after examining the written feedback line by line carefully, repeatedly and independently. Any discrepancies in the major categories were discussed and resolved in a meeting. The qualitative data was used to supplement and explain the findings of pilot RCT.

\section{Results}

Data were collected from April 2018 to March 2019 in Hong Kong. Eighty-seven people were assessed for eligibility. Nine people were excluded as they did not meet the eligibility criteria for MetS after a physical assessment and one declined to participate in the study after its purpose was explained. Seventy-seven participants (38 experimental and 39 control participants) completed the study. Mean age was 57.42 (SD 6.43 ) and 60.45 (SD 7.49) for the App group and the control group. App group had $52.6 \%(\mathrm{n}=20)$ male participants while the control group had only $35.9 \%(n=14)$ male participants. Attrition rates of $5.3 \%(n=2)$ and $7.7 \%(n=3)$ were found at T2, whereas attrition rates of $13.16 \%(n=5)$ and $10.26 \%(n$ $=4$ ) were found at T3 for the app and booklet groups, respectively. The total attrition rate for all participants was $11.69 \%$ at T3. No significant difference was found in demographic data between those who dropped out and those who completed the study. Moreover, no significant differences were found in baseline demographic or outcome data between groups (Tables 1 and 2). The mean age of the participants was 58.95 years (standard deviation [SD], 7.1 years) and 44.2\% $(n=34)$ of participants were male. Most participants were obese, with a mean body mass index (BMI) of 27.02 (SD, 4.21) and a mean waist circumference of approximately $96 \mathrm{~cm}$ (SD, $10.35 \mathrm{~cm})$. Their mean systolic blood pressure was 133.17 $\mathrm{mmHg}$ (SD, $16.45 \mathrm{mmHg}$ ) and their mean diastolic blood pressure was $83.6 \mathrm{mmHg}$ (SD, $14.73 \mathrm{mmHg}$ ). Most participants had completed secondary education or higher $(84.4 \%$, $\mathrm{n}=65)$, were married $(71.43 \%, \mathrm{n}=55)$, were working fulltime or part-time $(53.25 \%, \mathrm{n}=41)$, and were not currently smoking regularly $(98.7 \%, \mathrm{n}=76)$. The majority $(80.5 \%$, $\mathrm{n}=62$ ) of participants had an exercise habit, but their mean exercise time was approximately $128 \mathrm{~min}$ (SD, $96 \mathrm{~min}$ ), which was less than the exercise time recommended by the WHO (150 min per week). Their exercise self-efficacy score was below average, at 37.14 (SD, 14.65). No significant difference was found between the two groups with respect to demographic or clinical characteristics (Table 2).

Table 3 summarizes the mean, SD of outcomes and effect size and Table 4 summarizes GEE results of outcomes (body weight, BMI, waist circumference, blood pressure, lipid and fasting blood glucose concentrations, total exercise time, GSLTPA [exercise amount], and exercise self-efficacy [SEE]) across three time points between the two groups. Compared to the booklet, the app has produced medium effect sizes on weight, GSLTPA score and SEE, small effect sizes on BMI, waist circumference, systolic blood pressure and fasting blood glucose and very small effect on the remaining outcomes. At T3 (3 months), the app group showed a significant reduction in body weight compared to the booklet group $(\beta=-1.069, p=0.012)$. BMI was also significantly different between groups with better performance by app group $(\beta=-0.371, p=0.026)$.

The app group showed a significant improvement in total exercise time compared with the booklet group at T2 (1 month; $\beta=54.476, p=0.003$ ), but not at T3 (3 months). In addition, the app group showed a significant improvement in the total amount of exercise (GSLTPA) at T2 $(\beta=5.93, p=0.029)$ and T3 $(\beta=8.45, p=0.038)$. A significant greater increase in exercise self-efficacy was also seen in the app group at T2 $(\beta=7.919, \mathrm{p}=0.002)$ and $\mathrm{T} 3(\beta=10.62, p=0.001)$ compared to booklet group. However, there was no significant interactive effect seen for the other outcomes (blood pressure, lipid and fasting glucose concentrations) at T3. 
Table I Demographic and Clinical Characteristics of Participants

\begin{tabular}{|c|c|c|c|c|}
\hline Participants' Characteristics & All (N=77) N (\%) & App Group ( $\mathbf{N = 3 8 ) ~ N ~ ( \% ) ~}$ & Booklet Group (N=39) N (\%) & p-value \\
\hline Age, mean (SD) & $58.95(7.10)$ & $57.42(6.43)$ & $60.45(7.49)$ & 0.061 \\
\hline Gender & & & & 0.139 \\
\hline Male & $34(44.2)$ & $20(52.6)$ & 14 (35.9) & \\
\hline Female & $43(55.8)$ & $18(47.4)$ & $25(64.1)$ & \\
\hline Height $(\mathrm{cm})$, mean $(\mathrm{SD})$ & $1.62(0.89)$ & $1.64(0.09)$ & $1.6(0.08)$ & $0.024 *$ \\
\hline Weight $(\mathrm{kg})$, mean $(\mathrm{SD})$ & $71.38(15.24)$ & $73.61(15.66)$ & $69.2(14.69)$ & 0.206 \\
\hline BMI, mean (SD) & $27.02(4.2 I)$ & $27.1(4.4 I)$ & $26.95(4.07)$ & 0.876 \\
\hline Waist $(\mathrm{cm})$, mean $(\mathrm{SD})$ & $96(10.35)$ & $95.89(9.87)$ & $96.11(10.92)$ & 0.926 \\
\hline Hip $(\mathrm{cm})$, mean (SD) & $100.94(7.3)$ & $101.24(7.42)$ & $100.65(7.27)$ & 0.725 \\
\hline Systolic blood pressure (mmHg), mean (SD) & $133.17(16.45)$ & $133.39(15.65)$ & $132.9(17.39)$ & 0.906 \\
\hline Diastolic blood pressure (mmHg), mean (SD) & $83.6(14.73)$ & $84.34(12.16)$ & $82.87(17)$ & 0.664 \\
\hline Heart rate, mean (SD) & $72(13.98)$ & $73.16(10.9)$ & $72.31(13.37)$ & 0.754 \\
\hline Fasting blood glucose (mmo/L), mean (SD) & $5.86(1.57)$ & $5.54(1.15)$ & $6.16(1.85)$ & 0.082 \\
\hline Total cholesterol (mmo/L), mean (SD) & $4.99(0.86)$ & $4.96(0.82)$ & $5.01(0.9)$ & 0.812 \\
\hline HDL cholesterol (mmo/L), mean (SD) & $1.39(0.34)$ & I.4I (0.34) & $1.37(0.36)$ & 0.669 \\
\hline LDL cholesterol (mmo/L), mean (SD) & $2.66(0.8)$ & $2.50(1.00)$ & $2.62(0.88)$ & 0.694 \\
\hline Education level & & & & $0.018^{*}$ \\
\hline Primary or below & $12(15.6)$ & $2(5.3)$ & $10(25.7)$ & \\
\hline Secondary & $46(59.7)$ & $22(57.9)$ & $24(61.5)$ & \\
\hline Tertiary & $19(24.7)$ & $14(36.8)$ & $5(12.8)$ & \\
\hline Marital status & & & & 0.621 \\
\hline Married & $55(78.3)$ & $29(76.3)$ & $26(66.7)$ & \\
\hline Not married & $9(21.7)$ & $4(10.5)$ & $5(12.8)$ & \\
\hline Separated/divorced/widow & $13(16.9)$ & $5(13.2)$ & $8(20.5)$ & \\
\hline Financial status & & & & 0.203 \\
\hline Good & $16(20.8)$ & II (29) & $5(12.8)$ & \\
\hline Average & $58(75.3)$ & $26(68.4)$ & $32(82.1)$ & \\
\hline Poor & $3(3.9)$ & I (2.6) & $2(5.1)$ & \\
\hline Residential status & & & & 0.591 \\
\hline Live alone & $14(18.2)$ & $6(16)$ & $8(20.5)$ & \\
\hline Live with family & $63(81.8)$ & $32(84)$ & $31(79.5)$ & \\
\hline Smoking & & & & 0.308 \\
\hline Yes & $\mathrm{I}(\mathrm{I} .3)$ & I (2.6) & $0(0)$ & \\
\hline No & $76(98.7)$ & $37(97.4)$ & $39(100)$ & \\
\hline Employment status & & & & 0.664 \\
\hline Full-time & $32(4 \mid .6)$ & $15(39.5)$ & $17(43.6)$ & \\
\hline Part-time & $9(11.7)$ & $6(15.8)$ & $3(7.7)$ & \\
\hline Housewife & $12(16.9)$ & $7(18.4)$ & $5(15.4)$ & \\
\hline Retired & $23(29.9)$ & $10(26.3)$ & $13(33.3)$ & \\
\hline Exercise habit & & & & 0.817 \\
\hline Yes & $62(80.5)$ & $31(81.6)$ & $31(79.5)$ & \\
\hline No & $13(19.5)$ & $7(18.4)$ & $8(20.5)$ & \\
\hline
\end{tabular}

Note: Significant * $p<0.05$. 
Table 2 Baseline Outcome Measures for Participants

\begin{tabular}{|c|c|c|c|c|}
\hline Baseline Measures & $\begin{array}{l}\text { All }(\mathbf{N}=77) \\
N(\%)\end{array}$ & $\begin{array}{l}\text { App Group }(\mathbf{N}=\mathbf{3 8}) \\
\mathbf{N}(\%)\end{array}$ & $\begin{array}{l}\text { Booklet Group }(\mathbf{N}=39) \\
\mathbf{N}(\%)\end{array}$ & p-value \\
\hline & Mean (SD) & Mean (SD) & Mean (SD) & \\
\hline Total exercise time per week Mean (SD) & $127.99(96.11)$ & $137.5(100.21)$ & $118.72(92.3)$ & 0.395 \\
\hline Godin weekly leisure time activity score (GSLTPA) Mean (SD) & $16.45(11.34)$ & $16.13(10.68)$ & $16.76(12.08)$ & $0.81 \mathrm{I}$ \\
\hline Exercise self-efficacy & $37.14(14.65)$ & 34.61 (13.26) & 39.61 (I5.67) & 0.134 \\
\hline
\end{tabular}

\section{Process Evaluation}

We assessed the feasibility of the program by examining the recruitment rate, which was $88.5 \%$, and the total attrition rate, which was $11.69 \%(n=9)$ at T3. Acceptability was assessed by a qualitative analysis of the perceptions of the participants. Most participants $(81.58 \% \mathrm{n}=31)$ in app group and $84.62 \%(n=33)$ of the booklet group appreciated the intervention program. The app group found that the app was useful for their exercise maintenance and that the information platform was particularly useful for their dietary choices and lifestyle modifications, such as increasing their amount of exercise. Suggested ways to further improve the app for exercise maintenance included adding a step counter $(\mathrm{n}=5)$ and more frequent reminder features $(n=2)$ to the app. Overall, these findings indicated that the proposed intervention was feasible and appreciated by the participants.

\section{Discussion}

The overall aim of this study was to examine the preliminary effect of a home-based interactive e-health educational intervention program for young-old clients with MetS, using either an app or a booklet to support their lifestyle modifications. There was no significant difference in clinical baseline demographic and outcome characteristics between the intervention and control groups. However, the total exercise time and exercise intensity of the participants at baseline were below the standard recommended by the $\mathrm{WHO}^{27}$ A significant interaction effect was observed for the primary outcome, body weight, and for BMI at T2 (3 months). These results indicated that the app effectively promoted regular aerobic physical exercise, such as brisk walking, leading to weight reduction within 3 months.

Two systematic reviews have highlighted that a successful structured e-health program should be specifically tailored to the target group and have features that support self-monitoring, feedback, the use of theory, interactivity, and goal setting to change behavior. ${ }^{16,38,39}$ Given that the same information on MetS was provided to the booklet group and to the app group, the members' area and interactive platform and other features of the MetS app may have further facilitated the self-monitoring of individual health and the recording of exercise, body weight, and waist circumference, thereby enhancing the participants' self-efficacy for adequate dietary control, exercise initiation, self-monitoring, and maintenance, leading to a greater amount of exercise and body weight reduction. ${ }^{29-31}$ In addition, the alert and reminder functions of the app encouraged the participants to maintain their exercise habits. ${ }^{22-24}$ Further, the results showed that the total amount of physical exercise, as measured by the GSLTPA score, significantly increased over the 3-month study period, indicating that the app was effective at promoting more moderate-intensity exercise to be performed. The intervention group also had significantly greater exercise self-efficacy than the control group over the 3-month study period.

Previous studies have consistently shown a positive effect of increased physical activity on body weight control and lipid profile over a period of 3 or more months. $^{13,22,23,39,40}$ The success of other programs $^{13,16,22,23,40}$ may be due to their labor-intensive components, such as individual counselling for dietary and exercise advice or a supervised exercise program. However, our study relied solely on the app support, leading to an increase in the amount of physical exercise and weight reduction over the 3-month study period. In this study, our intervention likely elicited short- to medium-term positive effects on physical exercise up to 3 months. Consistent with the preliminary RCT data, our qualitative findings of the process evaluation showed that the app was mostly accepted by the participants for support of their exercise maintenance. Our MetS App could be incorporated in the programme and promoted to the population on a larger scale to reduce MetS risks with limited human resource.

Lipid concentrations and blood pressure levels did not significantly differ between intervention groups, although 
Table 3 Mean and Standard Deviation of Outcomes

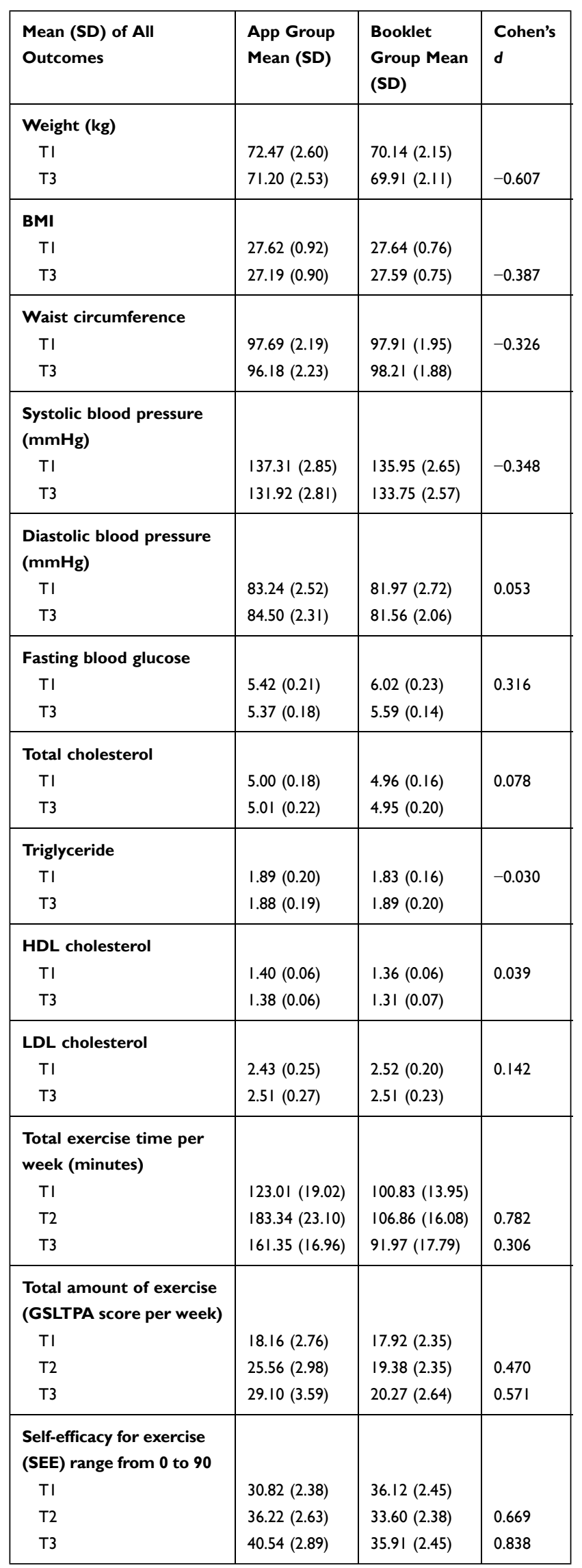

Table 4 Generalized Estimation Equation (GEE) Models for the Comparison of the Repeated Measures Outcome Variables Between the Two Groups

\begin{tabular}{|c|c|c|c|c|}
\hline \multirow[b]{2}{*}{ Weight (kg) } & \multirow[t]{2}{*}{$\beta$} & \multicolumn{2}{|c|}{$95 \% \mathrm{Cl}$} & \multirow[t]{2}{*}{$\mathbf{p}$} \\
\hline & & & & \\
\hline Group & 2.358 & -4.16 & 8.88 & 0.478 \\
\hline Month 3 & -0.217 & -0.66 & 0.23 & 0.337 \\
\hline Group*Month 3 & -1.069 & -1.90 & -0.24 & $0.012^{*}$ \\
\hline \multicolumn{5}{|l|}{ BMI } \\
\hline Group & -0.021 & -1.75 & 1.71 & 0.981 \\
\hline Month 3 & -0.06 & -0.27 & 0.16 & 0.620 \\
\hline Group*Month 3 & -0.371 & -0.70 & -0.04 & $0.026 *$ \\
\hline \multicolumn{5}{|l|}{ Waist (cm) } \\
\hline Group & -0.223 & -4.69 & 4.24 & 0.922 \\
\hline Month 3 & 0.296 & -1.23 & 1.82 & 0.703 \\
\hline Group*Month 3 & -1.801 & -4.26 & 0.66 & 0.151 \\
\hline \multicolumn{5}{|l|}{ Waist-hip ratio } \\
\hline Group & -0.003 & -0.03 & 0.23 & 0.834 \\
\hline Month 3 & 0.001 & -0.17 & 0.02 & 0.924 \\
\hline Group*Month 3 & -0.017 & -0.04 & 0.01 & 0.210 \\
\hline \multicolumn{5}{|l|}{ Systolic blood pressure } \\
\hline Group & 1.357 & -5.84 & 8.56 & 0.712 \\
\hline Month 3 & -2.199 & -5.60 & 1.20 & 0.205 \\
\hline Group*Month 3 & -3.185 & -9.19 & 2.82 & 0.299 \\
\hline \multicolumn{5}{|l|}{ Diastolic blood pressure } \\
\hline Group & 1.246 & -5.30 & 7.79 & 0.709 \\
\hline Month 3 & -0.403 & -5.00 & 4.20 & 0.864 \\
\hline Group*Month 3 & 1.843 & -4.36 & 8.05 & 0.560 \\
\hline \multicolumn{5}{|l|}{ Fasting blood glucose } \\
\hline Group & -0.596 & -1.32 & 0.12 & 0.105 \\
\hline Month 3 & -0.426 & -0.84 & -0.01 & $0.045^{*}$ \\
\hline Group*Month 3 & 0.374 & -0.12 & 0.87 & 0.140 \\
\hline \multicolumn{5}{|l|}{ Total cholesterol } \\
\hline Group & 0.039 & -0.36 & 0.44 & 0.850 \\
\hline Month 3 & -0.013 & -0.29 & 0.26 & 0.926 \\
\hline Group*Month 3 & 0.027 & -0.30 & 0.35 & $0.87 I$ \\
\hline \multicolumn{5}{|l|}{ Triglyceride } \\
\hline Group & 0.053 & -0.39 & 0.50 & 0.813 \\
\hline Month 3 & 0.060 & -0.24 & 0.36 & 0.692 \\
\hline Group*Month 3 & -0.064 & -0.47 & 0.34 & 0.756 \\
\hline \multicolumn{5}{|l|}{ HDL cholesterol } \\
\hline Group & 0.042 & -0.12 & 0.21 & 0.610 \\
\hline Month 3 & -0.05 & -0.22 & 0.12 & 0.556 \\
\hline Group*Month 3 & 0.022 & -0.15 & 0.20 & 0.801 \\
\hline \multicolumn{5}{|l|}{ LDL cholesterol } \\
\hline Group & -0.093 & -0.52 & 0.33 & 0.669 \\
\hline Month 3 & -0.009 & -0.25 & 0.23 & 0.942 \\
\hline Group*Month 3 & 0.089 & -0.34 & 0.52 & 0.686 \\
\hline
\end{tabular}


Table 4 (Continued).

\begin{tabular}{|l|c|c|c|c|}
\hline & $\beta$ & \multicolumn{2}{|c|}{$95 \%$ CI } & p \\
\hline $\begin{array}{l}\text { Total Exercise time per } \\
\text { week }\end{array}$ & & & & \\
Group & 22.312 & -23.87 & 68.50 & 0.344 \\
Month I & 6.026 & -16.09 & 28.14 & 0.593 \\
Month 3 & -8.858 & -46.21 & 28.50 & 0.642 \\
Group*Month I & 54.476 & 18.63 & 90.33 & $0.003^{* *}$ \\
Group*Month 3 & 47.377 & -10.60 & 105.23 & 0.067 \\
\hline Total amount of exercise & & & & \\
(GSLTPA score) & & & & \\
Group & 0.25 & -4.84 & 5.34 & 0.924 \\
Month I & 1.476 & -2.17 & 5.10 & 0.430 \\
Month 3 & 2.357 & -2.22 & 6.94 & 0.313 \\
Group*Month I & 5.933 & 0.59 & 11.27 & $0.029 *$ \\
Group*Month 3 & 8.584 & 0.72 & 16.45 & $0.032^{*}$ \\
\hline Self-efficacy for Exercise & & & & \\
(SEE) & & & & \\
Group & -5.294 & -11.94 & 1.35 & 0.119 \\
Month I & -2.522 & -6.47 & 1.43 & 0.211 \\
Month 3 & -0.208 & -4.08 & 3.66 & 0.916 \\
Group*Month I & 7.919 & 2.80 & 13.04 & $0.002^{* *}$ \\
Group*Month 3 & 10.62 & 4.09 & 15.77 & $0.001 * *$ \\
\hline
\end{tabular}

Notes: Time points (month I, and month 3 , with month 0 as reference), time points and groups interaction terms (group*month I, and group*month 3,) were showed for the GEE models. Variables adjusted in the GEE models: education. All statistical tests were two-sided and a $\mathrm{p}$-value $<0.05$ was considered statistically significant. Significant ${ }^{*} p<0.05, * * p<0.001$.

the triglyceride concentrations and systolic blood pressure were lower in the app group than the booklet group. The findings were consistent with previous similar studies in China that participants in the intervention group (with more health educational support) demonstrated continuous improvements in Blood pressure control, fasting blood glucose and blood lipid level, but no significant interaction effects were detected. ${ }^{12}$ This non-significant result may be attributed to the small sample size or short-term effects due to the limitations of the pilot study design. More studies might be required to confirm these outcomes.

This study has several limitations. First, the sample size was small and only two community centers were used for data collection, which may affect the generalizability of these results. Second, the data relied on participants' self-input of their exercise record and some participants may have forgotten to input their data. An objective tool for measuring exercise may be used in future studies, if funding allows. There are currently many activity-tracking devices on the market that can automatically record exercise and these could be used for such measurements. Furthermore, we made multiple comparisons in the hypothesis testing regarding the effectiveness of the app on the outcome variables, which have inflated the overall type 1 error, and large-scale main RCTs are needed to confirm the results observed in this pilot study.

Future research should involve a large-scale multicenter study. A sustainable, longer-term effect (6 months or longer) or a more intense form of e-health intervention with a stronger dose to engage the clients' exercise sustainability might be explored further in the future.

\section{Conclusion}

The MetS app, together with a lifestyle intervention programme, is a safe and feasible means of providing support to patients with MetS, to improve their exercise maintenance and help them with weight reduction. The MetS app developed in this study may be incorporated in the programme to support exercise maintenance and promote a healthy lifestyle in the community.

\section{Data Sharing Statement}

The authors do not intend to share individual deidentified participant data.

\section{Acknowledgments}

Sincere thanks to the Start-up Fund, the Hong Kong Polytechnic University for the groundwork and Health and Medical Research Fund, Food and Health Bureau of Hong Kong SAR to support our research. Special thanks to the clients who participate in our project.

\section{Disclosure}

The authors report no conflicts of interest in this study.

\section{References}

1. The International Diabetes Federation. The IDF consensus worldwide definition of the metabolic syndrome; 2017. Available from: https://www. idf.org/e-library/consensus-statements/60-idfconsensus-worldwidedefinitionof-the-metabolic-syndrome. Accessed December 15, 2020.

2. Mottillo S, Filion KB, Genest J, et al. The metabolic syndrome and cardiovascular risk a systematic review and meta-analysis. $J$ Am Coll Cardiol. 2010;56(14):1113-1132. doi:10.1016/j.jacc.2010.05.034

3. Ranasinghe P, Mathangasinghe Y, Jayawardena R, Hills A, Misra A. Prevalence and trends of metabolic syndrome among adults in the Asia-Pacific region: a systematic review. BMC Public Health. 2017;17(1):101. doi:10.1186/s12889-017-4041-1

4. Li R, Li WC, Lun ZJ, et al. Prevalence of metabolic syndrome in mainland China: a meta-analysis of published studies. BMC Public Health. 2016;16(1):296. doi:10.1186/s12889-016-2870-y

5. Ärnlöv J, Ingelsson E, Sundstrom J, et al. Impact of body mass index and the metabolic syndrome on the risk of cardiovascular disease and death in middle-aged men. Circulation. 2010;121(2):230-236. doi:10.1161/CIRCULATIONAHA.109.887521 
6. Saklayen MG. The global epidemic of the metabolic syndrome. Curr Hypertens Rep. 2018;20(2):12. doi:10.1007/s11906-018-0812-z

7. Bassi N, Karagodin I, Wang S, et al. Lifestyle modification for metabolic syndrome: a systematic review. Am J Med. 2014;127 (12):1242.e1-10. doi:10.1016/j.amjmed.2014.06.035

8. Kim CJ, Kim DJ, Park HR. Effects of a cardiovascular risk reduction intervention with psychobehavioral strategies for Korean adults with type 2 diabetes and metabolic syndrome. J Cardiovasc Nurs. 2011;26 (2):117-128. doi:10.1097/JCN.0b013e3181ec02ae

9. Mujica V, Urzúa A, Leiva E, et al. Intervention with education and exercise reverses the metabolic syndrome in adults. $J \mathrm{Am} S O \mathrm{C}$ Hypertens. 2010;4(3):148-153. doi:10.1016/j.jash.2010.02.008

10. Munakata M, Honma $\mathrm{H}$, Akasi $\mathrm{M}$, et al. Repeated counselling improves the antidiabetic effects of limited individualized lifestyle guidance in metabolic syndrome: j-STOP-METS final results. Hypertens Res. 2011;34(5):612-616. doi:10.1038/hr.2010.272

11. Nanri A, Tomita K, Matsushita Y, et al. Effect of six months lifestyle intervention in Japanese men with metabolic syndrome: randomized controlled trial. J Occup Health. 2012;54(3):215-222. doi:10.1539/ joh.11-0238-OA

12. Wang Q, Chair SY, Wong EM. The effects of a lifestyle intervention program on physical outcomes, depression, and quality of life in adults with metabolic syndrome: a randomized clinical trial. Int J Cardiol. 2016;230:461-467. doi:10.1016/j.ijcard.2016.12.084

13. World Health Organization. eHealth; 2017. Available from: http:// www.who.int/ehealth/en/. Accessed December 152020.

14. Azar KM, Koliwad S, Poon T, et al. The electronic CardioMetabolic Program (eCMP) for patients with cardiometabolic risk: a randomized controlled trial. J Med Internet Res. 2016;18(5):e134. doi: $10.2196 /$ jmir. 5143

15. Jahangiry L, Montazeri A, Najafi M, Yaseri M, Farhangi M. An interactive web-based intervention on nutritional status, physical activity and health-related quality of life in patient with metabolic syndrome: a randomized-controlled trial (the Red Ruby Study). Nutr Diabetes. 2017;7(1):e240. doi:10.1038/nutd.2016.35

16. Khaylis A, Yiaslas T, Bergstrom J, Gore-Felton C. A review of efficacious technology-based weight-loss interventions: five key components. Telemed J E Health. 2010;16(9):931-938. doi:10.1089/tmj.2010.0065

17. Weinstock RS, Brooks G, Palmas W, et al. Lessened decline in physical activity and impairment of older adults with diabetes with telemedicine and pedometer use: results from the IDEATel study. Age Ageing. 2011;40(1):98-105. doi:10.1093/ageing/afq147

18. Liebreich T, Plotnikoff RC, Courneya KS, et al. Diabetes NetPLAY: a physical activity website and linked email counselling randomized intervention for individuals with type 2 diabetes. Int J Behav Nutr Phys Act. 2009;6(1):18. doi:10.1186/1479-5868-6-18

19. Oh B, Cho B, Han MK, et al. The effectiveness of mobile phone-based care for weight control in metabolic syndrome patients: randomized controlled trial. JMIR mHealth uHealth. 2015;3(3):e83. doi:10.2196/mhealth. 4222

20. Wang J, Sereika SM, Chasens ER, et al. Effect of adherence to self-monitoring of diet and physical activity on weight loss in a technology-supported behavioral intervention. Patient Prefer Adherence. 2012;6:221-226. doi:10.2147/PPA.S28889

21. Jacobs N, De Bourdeaudhuij I, Claes N. Surfing depth on a behaviour change website: predictors and effects on behaviour. Inform Health Soc Care. 2010;35(2):41-52. doi:10.3109/17538157.2010.492922

22. LaPlante C, Peng W. A systematic review of e-health interventions for physical activity: an analysis of study design, intervention characteristics, and outcomes. Telemed E Health. 2011;17(7):509-523. doi:10.1089/tmj.2011.0013
23. Spring B, Duncan JM, Janke E, et al. Integrating technology into standard weight loss treatment: a randomized controlled trial. JAMA Intern Med. 2013;173(2):105-111. doi:10.1001/ jamainternmed.2013.1221

24. Whitehead L, Seaton P. The effectiveness of self-management mobile phone and tablet apps in long-term condition management: a systematic review. J Med Internet Res. 2016;18(5):e97. doi:10.2196/jmir.4883

25. Moher D, Hopewell S, Schulz KF, et al. CONSORT 2010 explanation and elaboration: updated guidelines for reporting parallel group randomised trials. Int J Surg. 2012;10(1):28-55.

26. Hertzog MA. Considerations in determining sample size for pilot studies. Res Nurs Health. 2008;31(2):180-191. doi:10.1002/nur.20247

27. Centers for Disease Control and Prevention (CDC). The benefits of physical activity; 2018. Available from: https://www.cdc.gov/physica lactivity/basics/pa-health/index.htm. Accessed December 30, 2020.

28. Lin CH, Chiang SL, Tseng WC, Chiang LC. Systematic review of impact of lifestyle-modification programs on metabolic risks and patient-reported outcomes in adults with metabolic syndrome. Worldviews Evid Based Nurs. 2014;11(6):361-368. doi:10.1111/wvn.12069

29. Janz N, Champion V, Strecher V. The health belief model. In: Glans K, Rimer B, Lewis F, editors. Health Behavior and Health Education - Theory, Research, and Practice. 3rd ed. New York: Jossey-Bass: John Wiley \& Sons Inc; 2002:45-63.

30. Bandura A. Self-Efficacy: The Exercise of Control. New York: W.H. Freeman; 1997.

31. Carlson JJ, Norman GJ, Feltz DL, Franklin BA, Johnson JA, Locke SK. Self-efficacy, psychosocial factors, and exercise behavior in traditional versus modified cardiac rehabilitation. $J$ Cardiopulm Rehabil. 2001;21 (6):363-373. doi:10.1097/00008483-200111000-00004

32. Godin G. The Godin-Shephard leisure-time physical activity questionnaire. Health Fit J Canada. 2011;4(1):18-22.

33. Godin G, Shephard RJ. A simple method to assess exercise behavior in the community. Can J Appl Sport Sci. 1985;10(3):141-146.

34. Wong EML, Leung DYP, Sit JWH, Chan AWK, Chair SY. Prospective validation of the Chinese version of the self-efficacy for exercise scale among middle-aged patients with coronary heart disease. Rehabil Nurs. 2020;45(2):74-79. doi:10.1097/RNJ.0000000000000156

35. Singer JD, Willett JB. Applied Longitudinal Data Analysis: Modeling Change and Event Occurrence. Oxford University press; 2003.

36. Sawilowsky S. New effect size rules of thumb. J Mod Appl Stat Methods. 2009;8(2):597-599. doi:10.22237/jmasm/1257035100

37. Sandelowski M. Combining qualitative and quantitative sampling, data collection, and analysis techniques in mixed method studies. Res Nurs Health. 2000;23(3):246-255. doi:10.1002/1098-240X (200006)23:3<246::AID-NUR9>3.0.CO;2-H

38. Whelton PK, Carey RM, Aronow WS, et al. 2017 ACC/AHA/AAPA/ ABC/ACPM/AGS/APhA/ASH/ASPC/NMA/PCNA guideline for the prevention, detection, evaluation, and management of high blood pressure in adults: a report of the American College of Cardiology/American Heart Association task force on clinical practice guidelines. Hypertension. 2018;71(6):e13-e115. doi:10.1161/HYP.0000000000000065

39. Cho SMJ, Lee JH, Shim JS, et al. Effect of smartphone-based lifestyle coaching app on community-dwelling population with moderate metabolic abnormalities: randomized controlled trial. $J$ Med Internet Res. 2020;22(10):e17435. doi:10.2196/17435

40. Luley C, Blaik A, Götz A, et al. Weight loss by telemonitoring of nutrition and physical activity in patients with metabolic syndrome for 1 year. J Am Coll Nutr. 2014;33(5):363-374. doi:10.1080/ 07315724.2013.875437 


\section{Publish your work in this journal}

Clinical Interventions in Aging is an international, peer-reviewed journal focusing on evidence-based reports on the value or lack thereof of treatments intended to prevent or delay the onset of maladaptive correlates of aging in human beings. This journal is indexed on PubMed Central, MedLine, CAS, Scopus and the Elsevier
Bibliographic databases. The manuscript management system is completely online and includes a very quick and fair peer-review system, which is all easy to use. Visit http://www.dovepress.com/ testimonials.php to read real quotes from published authors. 